\title{
THE MOTION OF PLUTO OVER THE AGE OF THE SOLAR SYSTEM
}

\author{
HIROSHI KINOSHITA AND HIROSHI NAKAI \\ National Astronomical Observatory \\ 2-21-1 Osawa, Mitaka, Tokyo, Japan \\ E-mail(internet):Kinoshita@c1.mtk.nao.ac.jp
}

\begin{abstract}
Pluto's motion is chaotic in the sense that the maximum Lyapunov exponent is positive and the Lyapunov time (the inverse of the Lyapunov exponent) is about 20 million years (Myr). We have carried out the numerical integration of Pluto over the age of the solar system (5.7 billion years towards the past and 5.5 billion years towards the future), which is about 280 times of the Lyapunov time. Our integration does not show any indication of gross instability in the motion of Pluto. The time evolution of Keplerian elements of a nearby trajectory of Pluto at first grow linearly with the time and then start to increase exponentially. These exponential divergences stop at about $420 \mathrm{Myr}$ and saturate. The exponential divergences are suppressed by the following three resonances that Pluto has:

(1) Pluto is in the 3:2 mean motion resonance with Neptune and the libration period of the critical argument is about 20000 years.

(2) The argument of perihelion librates around 90 degrees and its period is 3.8 Myr.

(3) The motion of the Pluto's orbital plane referred to the Neptune's orbital plane is synchronized with the libration of the argument of perihelion (a secondary resonance). The libration period associated with the second resonance is $34.5 \mathrm{Myr}$.

We briefly discuss the motions of Kuiper belt objects in a 3:2 mean motion resonance with Neptune and several possible scenarios how Pluto evolves to the present stable state.
\end{abstract}




\section{Numerical Exploration of Pluto's motion}

Pluto was found on January 21,1930 by Clyde W. Tombaugh of Lowell Observatory. Since the discovery Pluto moves only one third of her orbit. From this limited observation we discuss the orbital motion over the age of the solar system.

Cohen and Hubbard 1965 carried out numerical integration of outer planets over 120000 years and found Pluto is locked in the 3:2 mean motion resonance with Neptune. The critical argument of this resonance $\left(\theta_{1}=\right.$ $\left.3 \lambda_{p}-2 \lambda_{n}-\varpi_{p}\right)$ librates around 180 degrees and its period is about 20000 years and its amplitude is 76 degrees. The libration means that Neptune passes Pluto near at her aphelion, it prevents a close approach of Pluto to Neptune and keeps Pluto at least 16 AU. from Neptune. Their results show that secular like change in the eccentricity and inclination of Pluto.

Next important investigation on long-period behavior of Pluto was made by William and Benson 1971. They integrated over 4.5 Myr the averaged equations of Pluto's motion disturbed by Jupiter, Saturn, Uranus, and Neptune whose motions are taken from the secular perturbation theory by Brouwer and van Woerkom 1950. Their treatment is equivalent to the first-order secular perturbation.

They found the argument of Pluto's perihelion $\left(\theta_{2}=\varpi_{P}-\Omega_{P}\right)$ librates about 90 degrees with an amplitude of 24 degrees and a period of about 4 Myr. The secular like changes in the eccentricity and the inclination found in Cohen and Hubbard 1965 are parts of this long-period motions. The libration of $\theta_{2}$ prevents Pluto's perihelion from getting close to the plane of the disturbing planets, especially to Neptune's orbital plane. The dominant periodic variations of the eccentricity and the inclination are synchronized with the libration of the argument of perihelion, which is qualitatively explained by the secular perturbation theory (Kozai 1962) by assuming an exact 3:2 mean motion resonance $\left(\theta_{1}=180\right.$ degrees).

Nacozy and Diehl 1972 and 1978 analytically investigated Pluto's motion with use of canonical perturbation method and confirmed the libration of the argument of pericenter discovered by Williams and Benson 1971.

Kinoshita and Nakai 1984 confirmed the libration of the argument of perihelion directly from the numerical integration over $5 \mathrm{Myr}$ of outer planets.

Based on the 4.5 Myr integration, William and Benson 1971 made the following two conjectures on Pluto's motion:

1)The circulation period of $\theta_{3}=\Omega_{P}-\Omega_{N}$ is equal to the libration period of the argument of perihelion.

2) $\theta_{4}=\varpi_{P}-\varpi_{N}+3\left(\Omega_{P}-\Omega_{N}\right)$ librates.

If these conjectures are correct, these effects increase the minimum distance 
between Pluto and Neptune and stabilize Pluto-Neptune system.

The first conjecture was confirmed by Milani et al. 1989 using Longstop, $100 \mathrm{Myr}$ numerical integrations of outer planets (Nobili et al. 1989). This type of resonance is called a 1:1 secondary resonance. The libration period associated with this secondary resonance is $34.5 \mathrm{Myr}$ in both Longstop and our 5.5 billion years integration of outer planets (Kinoshita and Nakai 1995), which is discussed in the next section.

The second conjecture was confirmed by Kinoshita and Nakai 1995 with use of the 5.5 billion years. The argument $\theta_{4}$ librates about 180 degrees with a period of $570 \mathrm{Myr}$.

The fact that the conjectures based on the first-order secular perturbation are confirmed may indicates that the planetary system in the age of the solar system could be investigated with use of averaged equations of motion. Laskar 1988 and 1989 discussed the long-term behavior of the planetary system (excluding Pluto) with use of the secular perturbation theory of second order with respect to the planetary masses and five degrees in eccentricity and inclination, which is higher by one order than Brouwer and van Woerkom's theory 1950. Sussman and Wisdom 1992 carried out 100 Myr integration of the whole planetary system including Pluto and the relativistic effect and their results in the timescale of $100 \mathrm{Myr}$ are in excellent agreement with the results by Laskar et al. 1992, which are obtained from the integration of the averaged equations of motion. However it is not clear that whether a chaotic behavior of the system could be discussed with use of averaged equations of motion.

\section{Pluto's motion is chaotic}

In 1988 a shocking news "Pluto's motion is chaotic" came from Sussman and Wisdom. Their orbital computation was carried out Digital Orrery which is a special purpose computer. The maximum Lyapunov characteristic exponent is positive. The Lyapunov time(the inverse of Lyapunov exponent) is about $20 \mathrm{Myr}$. During their $850 \mathrm{Myr}$ integration Pluto does not show any global change in the orbital elements. Then Sussman and Wisdom 1992 carried out an integration of the whole solar system including Pluto over $100 \mathrm{Myr}$. The evolution of Pluto in this integration is similar to that of Pluto found in $845 \mathrm{Myr}$ integration and this integration also does not give any indication of a gross instability in the motion of Pluto. According to Wisdom 1992, "Since the system is apparently chaotic, we cannot rule out the possibility of gross instability. Recall some chaotic asteroid trajectories have been seen to evolve chaotically for 100 Lyapunov times at low eccentricity and then suddenly jump to large eccentricity. It will be very interesting to see a number of integrations of the whole solar system for the 
age of the solar system and longer."

Then we carried out orbital computation of outer planets over the age of the solar system forward to 5.5 billion years (Kinoshita and Nakai 1995) and backward to 5.7 billion years. As an integrator we used a linear symmetric multistep integrator developed by Quinlan and Tremaine 1990, whose truncation errors do not produce secular errors in the energy and angular momentum. In order to reduce the effect due to the accumulation of round-off errors, we adopted mixed precision arithmetic: the mutual gravitational forces are computed in double precision and the evaluations of the integrator are done in quadruple precision.

Figure 1 shows the orbital motions of five outer planets over 11.2 billion years. The $\mathrm{x}$-axis in this figure is the direction of the perihelion of Pluto. Each planets in this figure are plotted at every $4 \times 10^{8}$ days $\simeq 1 \mathrm{Myr}$. All outer planets move regularly over the age of the solar system. Three resonances in Pluto's motion (1) the 3:2 mean motion resonance, 2) the libration of the argument of perihelion, and 3) the secondary resonance between $\theta_{2}$ and $\theta_{3}$, which are discovered from the previous shorter integrations, are well kept during 11.2 billion years.

Then the question is what Pluto's chaotic motion is in the time scale of the age of the solar system. We investigate the time evolution of the behavior of a nearby object of Pluto. At first the differences of the orbital elements between Pluto and its nearby orbit grow linearly with time and then grows exponentially and saturate. The exponential growth corresponds with the fact that the Lyapunov exponent is positive. The saturation in the semi-major axis (see Figure 2, A1) is related to the 3:2 mean motion resonance (see Figure 2, A2 and B). The saturated value $(0.31 \mathrm{AU}$. ) in the semi-major axis is just twice of the amplitude of the 3:2 resonance libration. The saturations in the eccentricity, the inclination, the argument of perihelion, and the longitude of node are related to both the libration of the argument of perihelion and the secondary resonance between $\theta_{2}$ and $\theta_{3}$ (see Kinoshita and Nakai 1995 for more detailed discussion).

We can say even though Pluto's motion is chaotic in the sense that Pluto's Lyapunov exponent is positive, Pluto does stay in very stable configuration, which are protected at least in the age of the solar system by the three resonances.

In relation with the positive Lyapunov exponent of Pluto, we computed a Lyapunov exponent of Pluto in the following five simplified models.

- A) a circular restricted three body problem in three dimensions.

- A1) Neptune's motion is circular.

- A2) Neptune's motion is elliptic. 

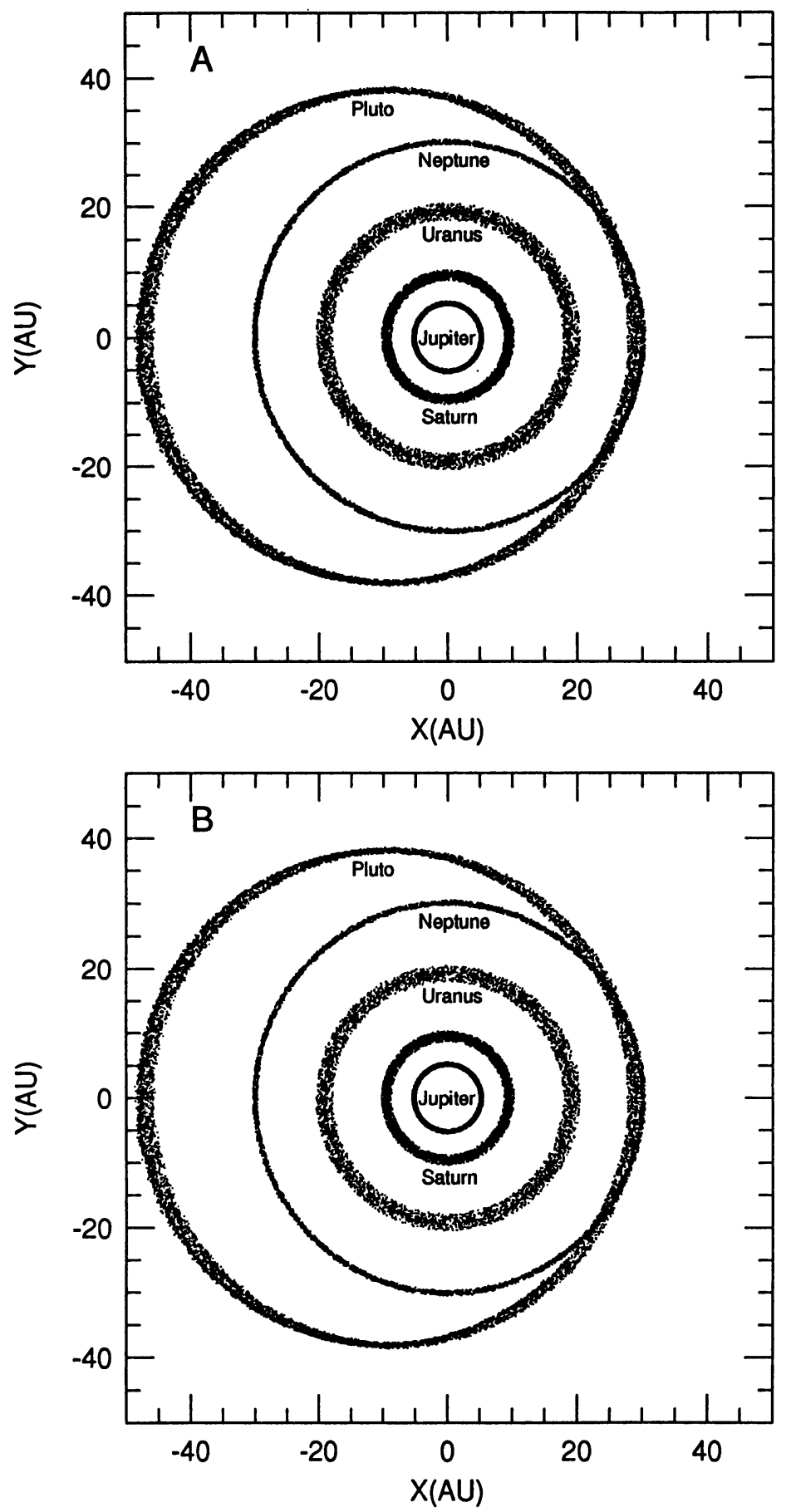

Figure 1. Orbital motions of outer planets over 11.2 billion years. The $x$-axis is the direction of Pluto's perihelion. A) past 5.7 billion years. B) future 5.5 billion years. 

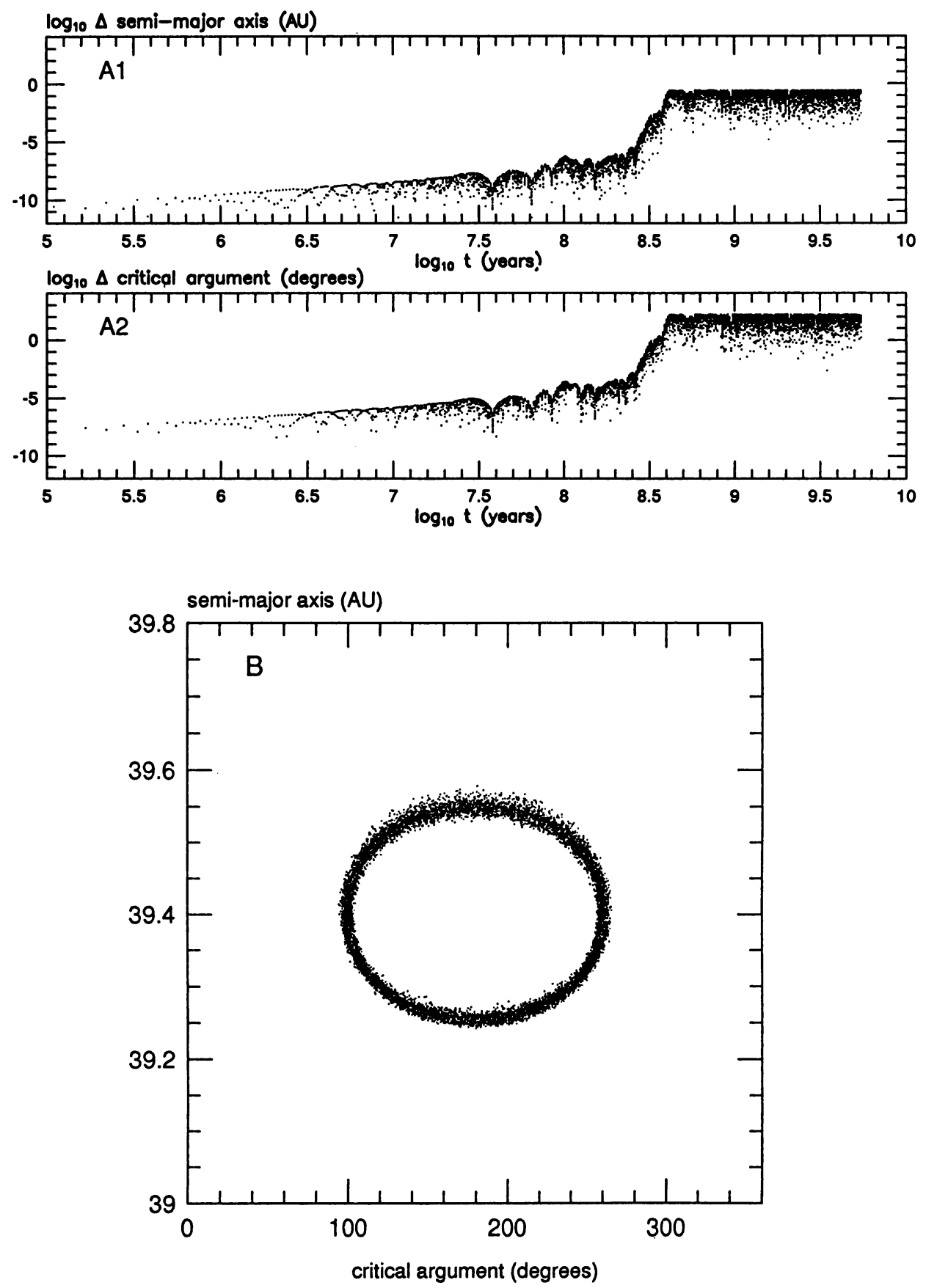

Figure 2. Time evolution of deviations of orbital elements between Pluto and its nearby orbit. A1)deviation of the semi-major axis. A2)deviation of the critical argument $\theta_{1}=3 \lambda_{p}-2 \lambda_{n}-\varpi_{p}$ B) The semi-major axis of Pluto versus the critical argument $\theta_{1}$. 
- B) The disturbing planets, Jupiter, Saturn, and Uranus, are treated as rings.

- B1) Neptune's orbit is elliptic in the ring plane and Pluto also moves in the ring plane.

- B2) Neptune's orbit is circular in the ring plane and Pluto's motion is three dimensional.

- B3) Neptune's orbit is elliptic in the ring plane and Pluto's motion is three dimensional.

The Lyapunov exponents in the models of A1, A2, B1, and B2 are zero and that of the model B3 is positive. The model B3 may be used to investigate the chaotic structure of Pluto's motion before attacking the chaotic structure of Pluto in more realistic model such as 6 body-problem. It is worthy to note that since the time evolution of a nearby orbit of Pluto is quite slow, a correct Lyapunov exponent cannot be obtained with use of a low arithmetic precision (double precision) due to an accumulation of round-off errors (Nakai et al. 1992).

Recently Ito (1995 private communication) has carried out 43 billion years integration of outer planets backwards, in which Pluto is not massless and the arithmetic is double precision and the integrator is a mixed variable symplectic integrator (Kinoshita et al. 1991). In this integration the first 5 billion years the three resonances Pluto has presently are all well kept but from about 10 billion years the motion of the argument of perihelion becomes irregular and finally the argument of perihelion circulates. At present we can not tell whether this transition is due to purely dynamical origin (a very slow process of diffusion) or originates from the round-off errors due to the adoption of double precision arithmetic.

\section{Kuiper-belt Objects in a 3:2 mean motion resonance}

Recently many trans-Neptunian objects (29 objects at this moment) or Kuiper belt objects have been detected and as many as 8 of 29 Kuiper belt objects may be locked in the 3:2 mean motion resonance with Neptune . In order to know whether the 3:2 mean motion resonance is conserved or not in longer timescale, we selected two objects,1993 SC that is located outside of Neptune's orbit and 1994TB that crosses Neptune's orbit.

The motion of 1993SC that does not cross Neptune's orbit is quite regular. We integrated this orbit over 1.3 billion years and the 3:2 mean motion resonance is kept and the orbit does not show any irregular behaviors. One of the interesting features of $1993 \mathrm{SC}$ is that the argument of perihelion is in a retrograde circulation.

The osculating eccentricity of $1994 \mathrm{~TB}$ is 0.37 and the orbit of $1994 \mathrm{~TB}$ crosses Neptune's orbit like Pluto does. Till 3 Myr the motion is quite regu- 
lar and the period of the critical argument of 3:2 mean motion resonance is about 17 thousands years. After about $3 \mathrm{Myr}$ the mean motion resonance is destroyed and then the orbit becomes chaotic. We integrated a nearby orbit whose initial distance in the phase space is different by $10^{-8}$ relatively from 1994TB. The 3:2 mean motion resonance in this nearby orbit is conserved over about $15 \mathrm{Myr}$ but then is destroyed. So far we could not identify the origin of the onset of this irregular motion. At least we could say this is not caused by the close approach to Uranus,even the minimum distance to Uranus is shorter than the distance to Neptune. After the destruction of the resonance, 1994TB comes to close to Uranus and then the orbit 1994TB begins to change drastically. The osculating elements of 1994TB adopted here are determined by assuming the 3:2 mean motion resonance (Minor Planet Electronic Circular (MPEC) 1994-E11). According to MPEC 1995M07, by adding new observations a 4:3 mean motion resonance for 1994TB is possible but the possibility of a 3:2 libration is not excluded and further observations are needed in order to distinguish the 4:3 and 3:2 solutions.

Other than the $3: 2$ resonance objects, the candidates in a 4:3, 5:3, and 2:1 resonances with Neptune have been detected. It is necessary to investigate the resonance structure in the Kuiper belt and the dynamical similarity or difference from the resonance structure of the main asteroidal belt, which are studied by Yoshikawa 1989, 1990, and 1992, Morbidelli and Moons 1993, and Moon and Morbidelli 1995.

\section{Origin of Pluto}

As we have seen in the previous numerical simulations, Pluto is protected from global chaos by the three resonances, especially the 3:2 mean motion resonance and the libration of the argument of perihelion. So how Pluto evolves into the present state with three resonance lockings is a very interesting and challenging problem. There are many scenarios on the origin of Pluto, which may be divided in two categories, one type assumes a catastrophic event such as a close encounter or encounters and one type assumes a resonance sweeping. The scenarios of an encounter model are proposed by Lyttleton 1936 (an encounter between Pluto as a satellite and Triton), Harrington and van Flandern 1979 (a single encounter of Neptune with a massive solar system body), Dormand and Wolfson 1980 (an extension of Lyttleton hypothesis), Olsson-Steel 1988 (close encounters between Pluto as a planet and Neptune), and Levison and Stern 1995 (encounters between a planet Pluto and primordial Kuiper belt objects).

Resonance sweeping scenarios are proposed by Colombo and Franklin 1970 (evolution in the presence of a resisting medium whose density decreases with time) and recently Malhotra 1993 and 1995 (Pluto is trapped 
into the 3:2 mean motion resonance by Neptune whose semi-major axis is increasing).

Levison and Stern 1995 showed that with use of numerical simulations Pluto with a nearly circular and low inclined orbit placed at near the 3:2 mean motion resonance with Neptune could evolve to the present highly eccentric and inclined orbit due to purely gravitational interactions of the giant planets in the present configuration in a timescale about $10^{7}$ years or longer. This amplification of the eccentricity and inclination could push Pluto into the state of the 3:2 mean motion resonance and the libration of the argument of perihelion. However the amplitude of the critical argument $\theta_{1}$ is too large. In order to reduce the amplitude for the stabilization of Pluto's motion Levison and Stern propose two different scenarios: 1) collisional and gravitational scattering between planet Pluto and small neighboring bodies in the Kuiper belt or 2) a single, inelastic, giant collision.

According to Malhotra's scenario 1993 and 1995, at the early stage of the solar system, when planets are forming from planetesimals, Neptune's orbit gradually expands by getting angular momentum from planetesimals. Pluto at this stage was near circular and low inclined. Neptune's orbit gradually expands and when Neptune reaches at the place where the 3:2 mean motion is possible, and Pluto is captured in a 3:2 mean motion resonance and is kept in the resonance after this capture. The argument of perihelion, however, is not locked and hops between 90 and 270 degrees over 10 Myr timescales.

Her scenario is very attractive but her scenario bases on the assumption that Neptune's orbit expands at planetary formation period. At present time there is no concrete and definite scenario on the evolution of a semimajor axis of outer planets at their formation period.

\section{References}

Brouwer, D. and van Woerkom, A. J. J. (1950) The Secular Perturbations of the Orbital Elements of the Principal Planets, Astron. Papers Am. Ephemeris. 13(Pt. 2), pp. 85-107.

Cohen, C. J. and Hubbard, E. C. (1965) Libration of the Close Approaches of Pluto to Neptune, Astron. J. 70, pp. 10-13.

Colombo, G. and Franklin, F. A. (1970) On the Evolution of the Solar System and the Pluto-Neptune Case, in Periodic Orbits, Stability and Resonances Giacaglia (ed.), Reidel Pub. Comp., pp. 328-331.

Dormand, J. R. and Woolfson, M. M. (1980) The Origin of Pluto, Mon. Not. R. astr. Soc. 193, pp. 171-174.

Harrington, R. S. and van Flandern, T. C. (1979) The Satellites of Neptune and the Origin of Pluto, Icarus. 39, pp. 131-136.

Kinoshita, H. and Nakai, H. (1984) Motions of the Perihelion of Neptune and Pluto, Celestial Mechanics. 34, pp. 203-217.

Kinoshita, H. , Yoshida, H. , and Nakai, H. (1991) Symplectic Integrators and Applications to Dynamical Astronomy, Celestial Mechanics. 50, pp. (59-71). 
Kinoshita, H. and Nakai, H. (1995) Long-Term Behavior of the Motion of Pluto over 5. 5 Billion Years, in The Small Bodies in the Solar System and their Interactions with Planets Rickman (ed.), Kluwer, in press.

Kozai, Y. (1962) Secular perturbation of asteroids with high inclination and eccentricity, Astron. J. 67, pp. 591-598.

Laskar, J. (1988) Secular Evolution of the Solar System over 10 Million Years, Astron. Astrophys. 198, pp. 341-362.

Laskar, J. (1989) A numerical experiment on the chaotic behavior of the solar system, Nature. 338, pp. 237-238.

Laskar, J. , Quinn, T. R. , and Tremaine, s. (1992) Confirmation of Resonant Structure in the Solar System, Icarus. 95, pp. 148-152.

Levison, H. F. and Stern, S. A. (1995) Possible Origin and Early Dynamical Evolution of the Pluto-Charon Binary, Icarus. 116, pp. 315-339.

Lyttleton, R. A. (1936) On the Possible Results of an Encounter of Pluto with the Neptunian System, Mon. Not. Roy. Astron. Soc. 97, pp. 108-115.

Malhotra, R. (1993) The Origin of Pluto's Peculiar Orbit, Nature. . 365, pp. 819-821.

Malhotra, R. (1995) The Origin of Pluto's Orbit:Implications for the Solar System beyond Neptune, Astron. J. 110, pp. 420-429.

Milani, A. , Nobili, A. M. and Carpino, M. (1989) Dynamics of Pluto, Icarus. 82, pp. 200-217.

Morbidelli, A. and Moons, M. (1993) Secular Resonances in Mean Motion Commensurabilities: The $2 / 1$ and $3 / 2$ Cases, Icarus. 102, pp. 316-332.

Moons, M. and Morbidelli, A. (1995) Secular Resonances in Mean Motion Commensurabilities: The 4/1,3/1, 5/2, and 7/3 Cases, Icarus. 114, pp. 33-50.

Nacozy, P. E. and Diehl, R. D. (1972) On the Long-Term Motion of Pluto, Celestial Mechanics. 8, pp. 445-454.

Nacozy, P. E. and Diehl, R. D. (1978) A Discussion of the Solution for the Motion of Pluto, Celestial Mechanics. 17, pp. 405-421.

Nakai, H. , Kinoshita, H. , and Yoshida, H. (1992) Dependence on computer's arithmetic precision in calculation of Lyapunov characteristic exponent, in Proceedings of 25 the Symposium on Celestial Mechanics Kinoshita, H. and Nakai, H (eds.), pp. 1-10.

Nobili, A. M. , Milani, A. and Carpino, M. (1989) Fundamental frequencies and small divisors in the orbits of the outer planets, Astron. Astrophys. 210, pp. 313-336.

Olsson-Steel, D. I. (1988) Results of Close Encounters between Pluto and Neptune, Astron. Astrophys. 195, pp. 327-330.

Quinlan, D. and Tremaine, S. (1990) Symmetric multistep methods for the numerical integration of planetary orbits, Astron. J. 100, pp. 1694-1700.

Sussman, G. J. and Wisdom, J. (1988) Numerical evidence that the motion of Pluto is chaotic, Science. 241, pp. 433-437.

Sussman, G. J. and Wisdom, J. (1992), Chaotic evolution of the solar system, Science. 257, pp. 56-62.

Williams, J. G. and Benson, G. S. (1971) Resonances in the Neptune-Pluto system, Astron. J. 76, pp. 167-177.

Wisdom, J. (1992) Long term evolution of the solar system, in Chaos, Resonance and Collective Dynamical Phenomena in the Solar System S. Ferraz-Mello (ed.), Kluwer, pp. 17-24.

Yoshikawa, M. (1989) A Survey on the Motion of Asteroids in Commensurabilities with Jupiter, Astron. Astrophys. 213, pp. 436-458.

Yoshikawa, M. (1990) Motions of Asteroids at the Kirkwood Gaps. I. On the 3:1 resonance with Jupiter, Icarus. 87, pp. 78-102.

Yoshikawa, M. (1990) Motions of Asteroids at the Kirkwood Gaps. II. On the 5:2, 7:3, and 2:1 resonance with Jupiter, Icarus. 92, pp. 94-117. 